\title{
Editorial
}

\section{Multiport Technology: New Perspectives and Applications}

\author{
Serioja Ovidiu Tatu, ${ }^{1}$ Adriana Serban, ${ }^{2}$ Alexander Koelpin, ${ }^{3}$ and Mohamed Helaoui ${ }^{4}$ \\ ${ }^{1}$ Institut National de la Recherche Scientifique-Energie, Matériaux et Télécommunications, \\ 800 rue de la Gauchetière Ouest, Montréal, Québec, Canada \\ ${ }^{2}$ Department of Science and Technology, Linköping University, Bredgatan 34, SE-601, 74 Norrkoping, Sweden \\ ${ }^{3}$ Institute for Electronics Engineering, University of Erlangen-Nuremberg, Cauerstr. 9, 91058 Erlangen, Germany \\ ${ }^{4}$ Intelligent RF Radio Technology Laboratory (iRadio Lab), Department of Electrical and Computer Engineering, \\ Schulich School of Engineering, University of Calgary, Calgary, AB, 91058 Erlangen, Canada T2N 1N4
}

Correspondence should be addressed to Serioja Ovidiu Tatu; tatu@emt.inrs.ca

Received 13 April 2014; Accepted 13 April 2014; Published 7 July 2014

Copyright (C) 2014 Serioja Ovidiu Tatu et al. This is an open access article distributed under the Creative Commons Attribution License, which permits unrestricted use, distribution, and reproduction in any medium, provided the original work is properly cited.

\section{Introduction}

The multiport circuit theory was initially developed in the 1970 s by scientists for accurate automatized measurements of the complex reflection coefficients, in microwave network analysis [1-3]. These multiport pioneers highlighted its usefulness in microwave low-cost circuit characterizations (Sparameters).

Since 1994, the multiport techniques were further developed for microwave and millimeter-wave radios [4-6]. Until today, several multiport architectures for specific applications, such as communication transceivers [7-17], radar sensing [18-23], direction of arrival estimation [24-26], or phase noise measurements [27], have been developed and implemented.

Basically, the multiport is a passive circuit composed of several couplers interconnected by transmission lines and phase shifters. Its specific architecture and design are strongly related to the target application and the operating frequency. The multiport acts as an interferometer; its output signals are linear combinations of phase shifted input signals. By using the appropriate circuit design and appropriate devices connected to the output ports, this circuit can provide specific parameters, such as reflection coefficient, distance or modal measurements, phase and frequency analysis, quadrature down-conversion, or direct modulation of microwave/millimeter-wave frequencies.

As originally designed for automated measurements of the complex reflection coefficient, the multiport has a local oscillator input, a measurement port, and four outputs [3]. One of the outputs is used as a reference power level and powers measured at the other ones are function of the complex coefficient of the device under test connected to the measurement port. There are three different reflection coefficient values named $q_{i}$ points, which minimize the power at the corresponding $i$ output. The ideal architecture requests that $q_{i}$ points are to be spaced by $120^{\circ}$ and located equidistant from the origin of the complex plane.

The new application fields require a different architecture of the circuit and specific modules to be connected at its ports. The S-parameter matrix of the multiport circuit reveals that there are two clusters of ports, 1 to 4 and 5 and 6 . Inside each cluster, all the ports are perfectly matched and isolated, one versus the others [15]. In all applications they play separate functions, such as four outputs and, respectively, two RF inputs for down-conversion or four control inputs and RF output/input for direct modulators. If the $\mathrm{S}$ matrix is further analyzed, then it is straightforward that if four matched loads are connected to the first group of ports ( 1 to 4 ) and two RF signals are applied to other pair of ports (5 and 6), all output signals at first group of ports are function of both input signals of the second group. This is a fundamental difference, if compared to the multiport used in reflection coefficient measurements, where one of the outputs is used as a power reference [3]. The multiport has now four $q_{i}$ points spaced by $90^{\circ}$ multiples and located equidistant from the origin of the complex plane. The phase difference between the pair of odd 
$q_{i}$ points is $180^{\circ}$. The same result is obtained for the pair of even points [17].

The use of multiport technology in RF design is a good choice, especially if the operating frequency is in the high microwave or millimeter-wave range. The dimensions of the multiport circuit fabricated in miniature hybrid microwave integrated circuit (MHMIC) technology, usually around $1.5 \lambda_{g} \times 1.5 \lambda_{g}$, where $\lambda_{g}$ represents the guided wavelength, become small enough to be integrated on the same substrate with antennas [20]. Even if the multiport is further miniaturized, the antenna or array antenna size will determinate the final dimensions of a front end. The multiport circuit can be also used in the front-end design to operate at the frequencies where active components are not yet available in the market. In order to operate as demodulator or modulator, it requires only the use of power detectors or switches [28-32]. Therefore, research activities can be validated by front-end prototyping measurements, years before standard technologies become available.

This special issue highlights, through several examples and multiple references, some of the modern applications of the multiport technology and significant advances in fabrication procedures, in the recent years.

\section{Special Issue Papers}

This special issue dedicated to multiport technology hosts several papers covering last advances in six-port receivers, demodulators, radar sensing, and ultrawide band (UWB) phase noise measurements.

An interesting question in multiport technology is how many ports should be used to fulfill a given system specification. In their paper entitled "Performance of $2-3.6 \mathrm{GHz}$ five-port/three-phase demodulators with baseband analog I/Q regeneration circuit in direct-conversion receivers," $\mathrm{K}$. Abdou et al. compare the performance of a five-port (FPD), a three-phase (TPD), and a quadrature demodulator. First, the authors describe the basic principles of FPD and TPD. Unlike the FPD, that uses detectors for the down-conversion, the TPD multiplies the radio frequency (RF) and the local oscillator signal with the help of mixers. A baseband circuit for the analog $I / Q$ regeneration is designed to reduce the number of analog-to-digital converters from three to two and allows suppression of DC offset and second order intermodulation distortion (IMD2).

Finally, the implementation of all architectures is demonstrated; furthermore, detailed measurement results are presented. These results indicate that TPD outperforms FPD in terms of residual DC offset, IMD2, noise figure, and sensitivity as well as error vector magnitude. Moreover, TPD has advantages over quadrature demodulators in the case of $\mathrm{RF}$ carrier aggregation. Thus, the authors conclude that the presented TPD architecture is a potential candidate for future long term evolution (LTE-A) standard.

The use of the $60-\mathrm{GHz}$ band has attracted a great deal of interest over the last few decade, especially for its use in future compact transceivers dedicated to high-speed wireless applications in indoor environments $(57-64 \mathrm{GHz})$.
In this context, C. Hannachi et al., the authors of the paper "Complete characterization of novel MHMICs for V-band communication systems" show the characterization results of several new passive millimeter-wave circuits integrated on very thin ceramic substrate (125 microns, relative permittivity equal to 9.9). These components are designed for a complete integrated V-band six-port receiver front end, hosting an antenna array, low-noise amplifier, and quadrature demodulator.

The work is focused on the design and characterization of a novel rounded Wilkinson power divider, a $90^{\circ}$ hybrid coupler, a rat-race coupler, and a novel six-port circuit. The authors describe in detail the equipment, the calibration technique, and the multiple challenges in millimeter-wave measurements. A series of microphotographs show a typical fabricated ceramic die and details of all circuits, as prepared for probe station testing.

Measurement results prove that the proposed circuits are UWB components. The supplementary insertion losses, amplitude, and phase unbalancements are considered more than acceptable to build modulators/demodulators for modulation schemes having up to 16 symbols (BPSK to 16 QAM, PSK, or dual star). Keeping in account the $7 \mathrm{GHz}$ bandwidth allowed for V-band communication systems, the data rates can reach quasioptical values.

Apart from its ease of use, simplicity of implementation, and low power requirements, which are due to the passive circuit implementation nature of six-port based receiver, other benefits drove interests in using six-port technology in receivers' design for applications using lower frequencies than millimetre wave. Indeed, the wide frequency bandwidth coverage, configurability, and low cost are some of the additional features that make this architecture very attractive for the implementation of the software defined radio (SDR) concept in the wireless communication frequency bands. The utilization of six-port concept as a RF front end in communication receivers was investigated by A. Hasan and M. Helaoui; in their paper "Performance driven six-port receiver and its advantages over low-IF receiver architecture" the authors provide a comprehensive analysis of the performance of six-port-based receiver in terms of signal quality, dynamic range, noise figure, isolation, bandwidth, and cost. They compare these metrics to a conventional low-IF architecture performance. On one side, the wide RF frequency bandwidth available in six-port circuitry is a major advantage that allows for use in ultrawideband and multiband configuration, reconfigurability, and, therefore, suitability for SDR applications. On the other side, signal quality measured in terms of receiver noise figure, sensitivity, and dynamic range is the main limitation in such topology. The main component limiting the signal quality is the dynamic range of power detectors. By driving the power detectors beyond their square law region, the dynamic range can be extended at a cost of linearity degradation. The use of postcompensation and calibration technique can compensate this degradation, which results in configurable receivers with good linearity and dynamic range performance. A. Hasan and M. Helaoui showed that by using such postcompensation techniques, error vector magnitudes (EVMs) less than $1 \%$ can be obtained 
for complex modulated signals suggesting that such topology can be a very good candidate for an SDR receiver front end.

Besides the broad range of applications already introduced in this paper, another very promising topic is ranging and direction finding. In particular for industry grade distance measurements, the six-port operated as a microwave interferometer shows extraordinary performance, compared to common radar architectures.

In the paper entitled "A compact, versatile six-port radar module for industrial and medical applications," S. Linz et al. demonstrate that the six-port receiver used as continuous wave radar is suitable for a variety of application scenarios ranging from distance and vibration measurements in industrial environments to heartbeat detection for medical applications.

The authors present a compact $24 \mathrm{GHz}$ six-port radar sensor with reliable high-integration of all subcomponents in one single module. This sensor has been developed in cooperation with industry. After a general system overview and the theoretical description of the implemented six-port network, a short introduction to the digital signal processing strategy is given. Simulation results of the subcomponents are furthermore validated by measurements. The demonstrated results of the system's performance show a maximum relative error of $\pm 400 \mu \mathrm{m}$ and high precision in the micrometer range $( \pm 40 \mu \mathrm{m})$.

J. M. Ávila-Ruiz et al. propose in their paper new sixport for low-power oscillator phase noise measurements. This paper entitled "Six-port based architecture for phase noise measurement in the UWB band" demonstrates, on one side, the advances of the multiport technology that continues to be used as a reliable, accurate, low-power core of automated measurement setups. On the other side, it reflects the advances in development of the analytical theory, supporting and explaining the multiport technology and its large field of applications.

The presented phase noise measurement system combines two phase noise measurement approaches, that is, the delay line discriminator method and the phase noise measurement method based on the quadrature $I / Q$ six-port demodulator. The proposed solution takes advantage of the ability of the multiport demodulator to accurately handle low-power input signals and to detect useful signal magnitude and phase information. In addition, the delay line discriminator eliminates the need for expensive reference oscillator, as opposed to traditional phase noise measurement systems.

After briefly introducing the state-of-the-art phase noise measurements, the authors provide a detailed theoretical analysis that demonstrates the complex processing of signals performed by the multiport system. It is shown that the required phase noise information is embedded in the quadrature $I / Q$ components of the down-converted signal. To assess the intrinsic limitations of the system, internal noise sources are analysed and solutions are proposed to increase measurement sensitivity. Finally, phase noise measurements performed with the prototype are compared to those obtained with a commercial signal source analyzer.
The results presented by J. M. Ávila-Ruiz et al. reconfirm the multiport technology in its original application proposed in the 1970s as an accurate, flexible measurement set-up based on interferometric signal processing.

\section{Conclusion}

In the last 40 years, since the multiport (six-port) circuit is used, dozens of architectures have been proposed. The operating frequencies, fabrication technologies, and applications do not cease to evolve. Through several well-chosen examples, the special issue highlights some of the multiport technology modern applications.

These papers and related references demonstrate that multiport technology is a good competitor of the conventional approaches, especially in the microwave and millimeter-wave frequencies, where circuit dimensions, closely related to the guided wavelength, are in the $\mathrm{cm}$ range or smaller. Broadband circuits can be designed to cover a wide frequency spectrum and to allow quasioptical data rates for wireless communication. Impressive resolution and high accuracy can be obtained in range or angle-of-arrival measurements. Calibration and impairment mitigation techniques can also be used to improve demodulation results, especially in low microwave spectrum, where analog to digital convertors (ADC) and digital signal processing (DSP) techniques are available today.

Future work targets, among others, improvements in multiport circuit design for each specific application, the increasing of the operating frequencies, and, why not, the use of multiport systems in $\mathrm{THz}$ frequency range.

Serioja Ovidiu Tatu Adriana Serban Alexander Koelpin Mohamed Helaoui

\section{References}

[1] S. B. Cohn and N. P. Weinhouse, "An automatic microwave phase measurement system," Microwave Journal, vol. 7, no. 2, pp. 49-56, 1964.

[2] G. F. Engen and C. A. Hoer, "Application of an arbitrary 6-port junction to power-measurement problems," IEEE Transactions on Instrumentation and Measurement, vol. 21, no. 4, pp. 470474, 1972.

[3] G. F. Engen, “The six-port reflectometer: an alternative network analyzer," IEEE Transactions on Microwave Theory and Techniques, vol. 25, no. 12, pp. 1075-1080, 1977.

[4] T. Hentschel, "The six-port as a communications receiver," IEEE Transactions on Microwave Theory and Techniques, vol. 53, no. 3, pp. 1039-1047, 2005.

[5] A. Koelpin, G. Vinci, B. Laemmle, D. Kissinger, and R. Weigel, "The six-port in modern society," IEEE Microwave Magazine, vol. 11, no. 7, pp. 35-43, 2010.

[6] G. Vinci, S. Lindner, F. Barbon, R. Weigel, and A. Koelpin, "Promise of a better position," IEEE Microwave Magazine, vol. 13, no. 7, pp. S41-S49, 2012.

[7] J. Li, R. G. Bosisio, and K. Wu, "Computer and measurement simulation of a new digital receiver operating directly at 
millimeter-wave frequencies," IEEE Transactions on Microwave Theory and Techniques, vol. 43, no. 12, pp. 2766-2772, 1995.

[8] S. O. Tatu, E. Moldovan, K. Wu, and R. G. Bosisio, "A new direct millimeter-wave six-port receiver," IEEE Transactions on Microwave Theory and Techniques, vol. 49, no. 12, pp. 2517-2522, 2001.

[9] S. O. Tatu, E. Moldovan, K. Wu, R. G. Bosisio, and T. A. Denidni, "Ka-band analog front-end for software-defined direct conversion receiver," IEEE Transactions on Microwave Theory and Techniques, vol. 53, no. 9, pp. 2768-2776, 2005.

[10] Y. Zhao, J.-F. Frigon, K. Wu, and R. G. Bosisio, "Multi(six)port impulse radio for ultra-wideband," IEEE Transactions on Microwave Theory and Techniques, vol. 54, no. 4, pp. 1707-1711, 2006.

[11] S. O. Tatu and E. Moldovan, "V-band multiport heterodyne receiver for high-speed communication systems," EURASIP Journal on Wireless Communications and Networking, vol. 2007, Article ID 34358, 7 pages, 2007.

[12] R. G. Bosisio, Y. Y. Zhao, X. Y. Xu et al., "New wave radio," IEEE Microwave Magazine, vol. 9, no. 1, pp. 89-100, 2008.

[13] A. Serban, J. Östh, O. M. Karlsson, S. Gong, J. Haartsen, and P. Karlsson, "Six-port transceiver for $6-9 \mathrm{GHz}$ ultrawideband systems," Microwave and Optical Technology Letters, vol. 52, no. 3, pp. 740-746, 2010.

[14] C. de la Morena-Álvarez-Palencia and M. Burgos-Garcia, "Four-octave six-port receiver and its calibration for broadband communications and software defined radios," Progress in Electromagnetics Research, vol. 116, pp. 1-21, 2011.

[15] S. O. Tatu, E. Moldovan, and S. Affes, "Multi-port front-end and transceivers for V-band multi-gigabit/s communication systems," in Digital Front-End in Wireless Communications and Broadcasting Circuits and Signal Processing, pp. 707-732, Cambridge University Press, Cambridge, UK, 2011.

[16] A. Hasan and M. Helaoui, "Novel modeling and calibration approach for multi-port receivers mitigating system imperfections and hardware impairments," IEEE Transactions on Microwave Theory and Techniques, vol. 60, no. 8, pp. 2644-2653, 2012.

[17] T. Jiang, D. Hammou, C. Hannachi et al., "Six-port technology for millimeter-wave MIMO systems," in Proceedings of the European Radar Conference (EuRAD '13), pp. 279-282, Nuremberg, Germany, October 2013.

[18] E. Moldovan, S.-O. Tatu, T. Gaman, K. Wu, and R. G. Bosisio, "A new 94-GHz six-port collision-avoidance radar sensor," IEEE Transactions on Microwave Theory and Techniques, vol. 52, no. 3, pp. 751-759, 2004.

[19] B. Boukari, E. Moldovan, R. I. Cojocaru et al., "A 77-GHz sixport fmcw collision avoidance radar sensor with baseband analytical calibration," Microwave and Optical Technology Letters, vol. 51, no. 3, pp. 720-725, 2009.

[20] S. O. Tatu, B. Boukari, E. Moldovan, R. G. Bosisio, and K. $\mathrm{Wu}$, "Millimeter-wave multi-port radar sensor with integrated receiver front-end for automotive applications," in Proceedings of the IEEE MTT-S International Microwave Symposium Digest (MTT '12), pp. 1-3, Montreal, Canada, June 2012.

[21] F. Barbon, G. Vinci, S. Lindner, R. Weigel, and A. Koelpin, "A six-port interferometer based micrometer-accuracy displacement and vibration measurement radar," in Proceedings of the IEEE MTT-S International Microwave Symposium Digest (MTT '12), pp. 1-3, Montreal, Canada, June 2012.

[22] G. Vinci, S. Lindner, F. Barbon et al., "Six-port radar sensor for remote respiration rate and heartbeat vital-sign monitoring,"
IEEE Transactions on Microwave Theory and Techniques, vol. 61, no. 5, pp. 2093-2100, 2013.

[23] G. Vinci, S. Lindner, F. Barbon, S. Mann, R. Weigel, and A. Koelpin, "Six-Port microwave interferometer radar for mechanical vibration analysis," in Proceedings of the European Radar Conference (EuRAD '13), pp. 287-290, Nuremberg, Germany, October 2013.

[24] S. O. Tatu, K. Wu, and T. A. Denidni, "Direction-of-arrival estimation method based on six-port technology," IEE Proceedings: Microwaves, Antennas and Propagation, vol. 153, no. 3, pp. 263269, 2006.

[25] G. Vinci, A. Koelpin, F. Barbon, and R. Weigel, "Six-portbased direction-of-arrival detection system," in Proceedings of the Asia-Pacific Microwave Conference (APMC '10), pp. 18171820, December 2010.

[26] G. Vinci, F. Barbon, R. Weigel, and A. Koelpin, "A novel, wide angle, high resolution direction-of-arrival detector," in Proceedings of the 8th European Radar Conference (EuRAD '11), pp. 265-268, October 2011.

[27] S. O. Tatu, E. Moldovan, S. Affes, B. Boukari, K. Wu, and R. G. Bosisio, "Six-port interferometric technique for accurate $W$-band phase-noise measurements," IEEE Transactions on Microwave Theory and Techniques, vol. 56, no. 6, pp. 1372-1379, 2008.

[28] J. Östh, O. Owais, M. Karlsson, A. Serban, and S. Gong, "Schottky diode as high-speed variable impedance load in six-port modulators," in Proceedings of the IEEE International Conference on Ultra-Wideband (ICUWB '11), pp. 68-71, Bologna, Italy, September 2011.

[29] J. Östh, A. Serban, M. Karlsson, and S. Gong, "LO leakage in six-port modulators and demodulators and its suppression techniques," in Proceedings of the IEEE MTT-S International Microwave Symposium Digest (MTT '12), pp. 1-3, Montreal, Canada, June 2012.

[30] A. Serban, M. Karlsson, J. Östh, Owais, and S. Gong, "Differential circuit technique for six-port modulator and demodulator," in Proceedings of the IEEE/MTT-S International Microwave Symposium Digest (MTT '12), pp. 1-3, Montreal, Canada, June 2012.

[31] J. Östh, M. Karlsson, A. Serban, and S. Gong, "Carrier leakage suppression and EVM dependence on phase shifting network in six-port modulator," in Proceedings of the International Conference on Microwave and Millimeter Wave Technology (ICMMT '12), pp. 1-4, Shenzhen, China, May 2012.

[32] J. Östh, M. Karlsson, A. Serban, and S. Gong, "M-QAM six-port modulator using only binary baseband data, electrical or optical," IEEE Transactions on Microwave Theory and Techniques, vol. 61, no. 6, pp. 2506-2513, 2013. 

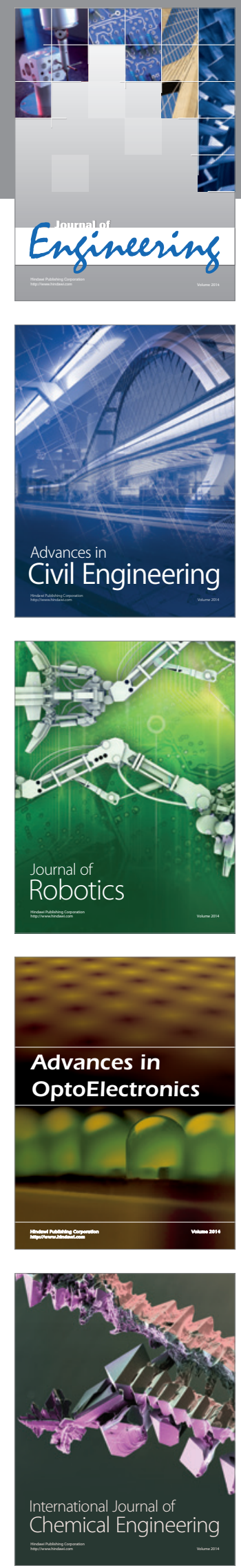

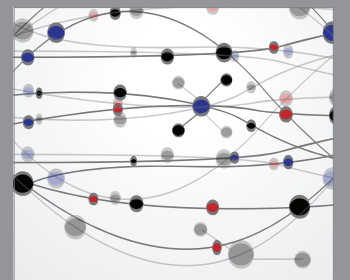

The Scientific World Journal
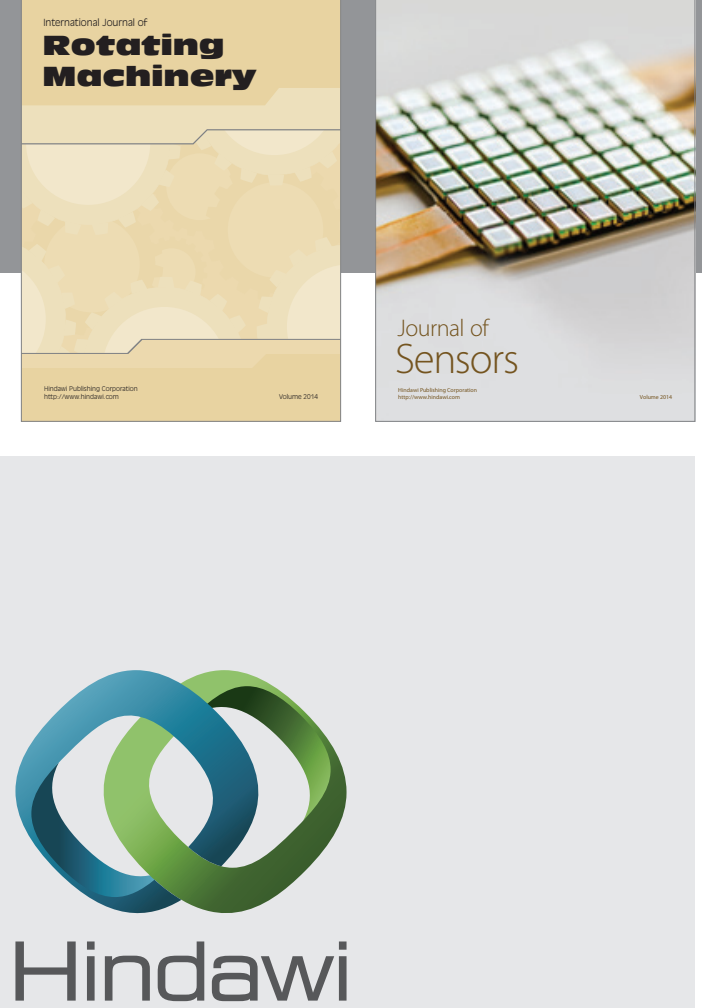

Submit your manuscripts at http://www.hindawi.com
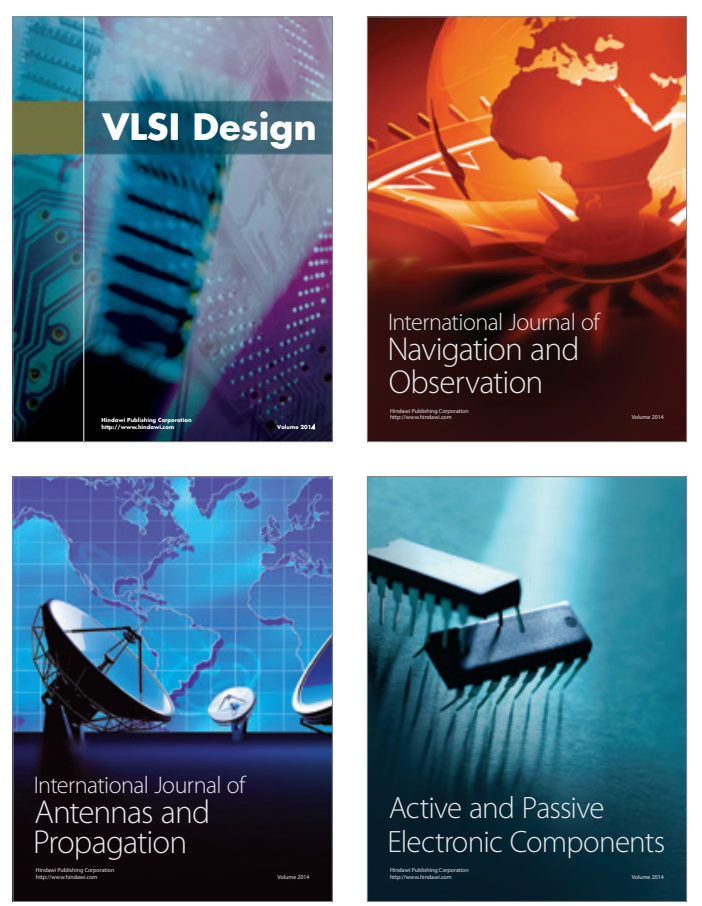
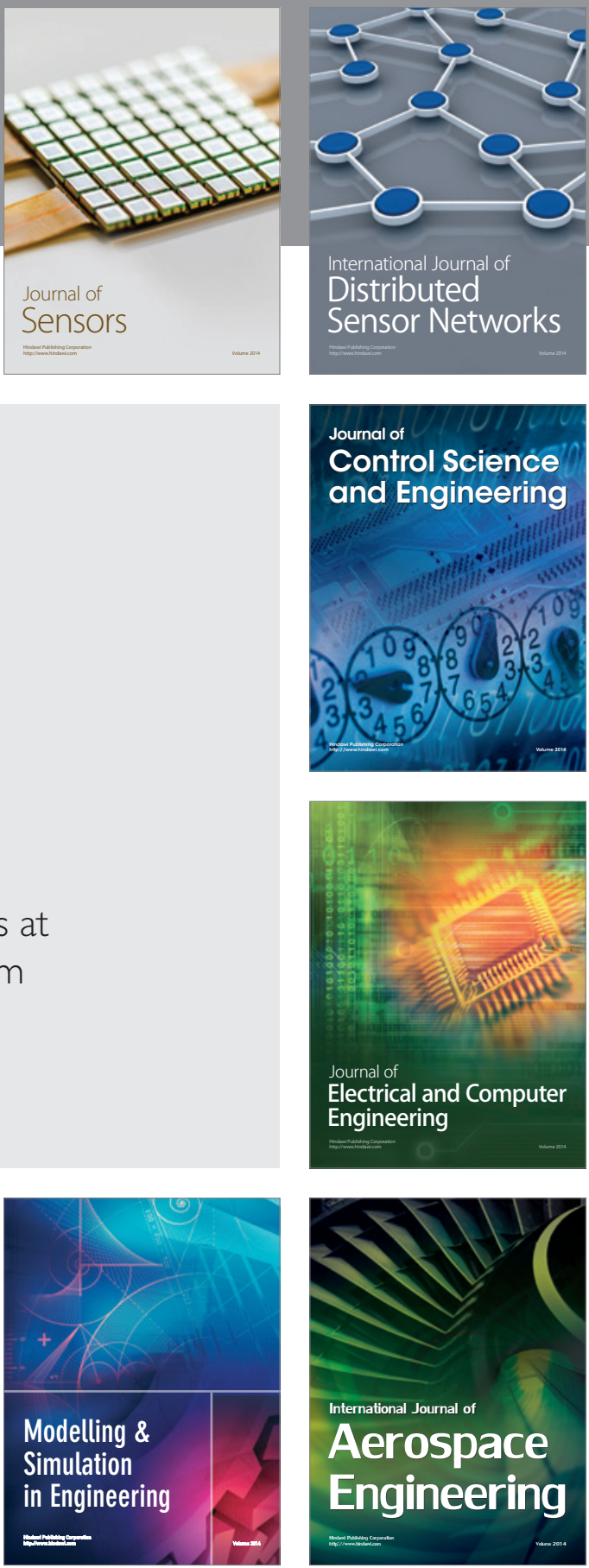

Journal of

Control Science

and Engineering
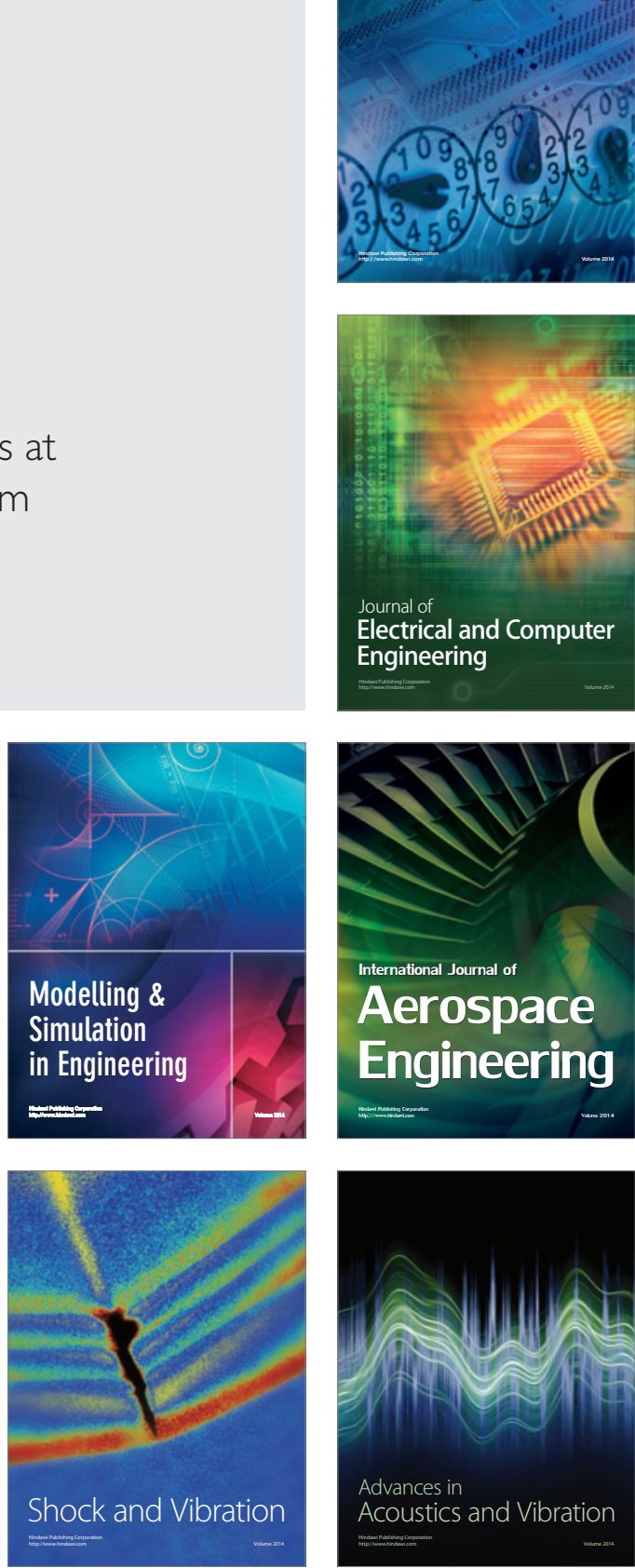